\title{
Simulation investigations of the coordinated traffic-responsive signal control strategy TUC with actuation at the local junction level
}

\author{
Diamantis Manolis, Christina Diakaki ${ }^{*}$, loannis Papamichail and Markos Papageorgiou
}

\begin{abstract}
Purpose: TUC (Traffic-responsive Urban Control), a strategy aiming at providing coordinated traffic-responsive signal control in large-scale urban networks, has demonstrated high efficiency, especially under saturated conditions. Nevertheless, as TUC adopts common cycle times for all junctions at any time, some green time waste may occur at links with low or strongly varying alternative demand, which could be mitigated by enabling local actuation. It is the aim of this paper to investigate potential benefits to TUC performance emerging from such local actuation.

Methods: Actuation may be performed in various ways: by relocating unused green time to stages serving high demand; or by preserving TUC-ordered offsets; or by combining as much as possible both of these features. To study and compare the performance and efficiency of these alternative ways to actuation under realistic traffic conditions, the AIMSUN microscopic simulation model of the road network of the city center of Chania, Greece, was used.

Results: Simulation investigations indicated relocating unused green time to stages serving high demand as the most promising way towards a TUC-coordinated locally-actuated control scheme. However, improvements over the TUC strategy by adopting this approach to actuation were moderate.

Conclusion: As the simulation results may be due to the signal control setup at the particular network under study and/or the examined demand scenarios, further investigations, including tests with other road networks and/or demand patterns are necessary before reaching any final conclusions concerning the best way to locally actuate the TUC-decided signal control plans.
\end{abstract}

Keywords: Traffic-responsive coordinated signal control, Traffic actuated signal control, Coordinated actuated signal control

\section{Introduction}

The usually limited availability of space in busy urban centers, along with the continuously increasing mobility requirements urge for solutions that will relieve from the serious congestion problems and their negative consequences for travel times, safety, fuel consumption, the environment and the quality of life in general. According to the White Paper on Transportation released by the European Commission (EC) in 2011 [1], the three main goals towards a sustainable transport system by 2050 are: "use less energy, use cleaner energy and better exploit

\footnotetext{
* Correspondence: cdiakaki@isc.tuc.gr

School of Production Engineering and Management, Technical University of Crete, University Campus, 73100 Chania, Greece
}

infrastructure". In the case of urban centers, such goals are best served by a variety of measures, such as shift to walking and cycling, increased use of public transport [2] and, last not least, by signal control strategies capable to adapt and respond automatically to the prevailing traffic conditions, tackling at the same time the problem of traffic flow control in a coordinated at network-wide level manner [3]. Systems such as SCOOT (Split, Cycle and Offset Optimization Technique) and SCATS (Sydney Co-ordinated Traffic Control System) are examples of such, commercially available, traffic-responsive systems, which were developed in the 70 s but are still adopted worldwide due to their benefits compared to fixed-time signal control [4]. 
The TUC (Traffic-responsive Urban Control) strategy studied herein is a traffic-responsive strategy developed in the late 90s, targeting particularly saturated traffic conditions, which are generally lacking adequate and efficient solutions [5]. TUC aims at the homogeneous utilization of the capacity of large-scale urban networks via the provision of coordinated traffic-responsive signal control settings. This objective has been approached by using appropriate methodological tools that allow for efficient application to large-scale networks and give rise to characteristics such as robustness, generality, simplicity and low computational effort [5].

The first field implementation and evaluation of the TUC strategy was undertaken within the European project TABASCO (Telematics Applications in BAvaria, SCotland and Others) for a part of the Glasgow urban network in Scotland [6]. TUC was subsequently applied to a part of the city center of Chania, Greece, under a national project funded by the Greek Ministry for Research and Technology [7]. The successful initial field implementations motivated a major inter-European EC co-funded effort, whereby TUC was implemented and evaluated in three urban networks with quite different traffic and control infrastructure characteristics [8]; the urban networks of Chania, Greece; Southampton, UK; and Munich, Germany. Later, TUC was also implemented in Macaé [9] and other cities in Brazil. More recently a new successful field implementation, incorporating novel strategy features, took place at the network of Chania, Greece [10].

TUC consists of three distinct but interconnected modules, which, at regular time intervals, produce traffic-adaptive settings for the signal control of a given network [11]. TUC adopts one common cycle time for all network junctions at any time. This common cycle time is computed considering the needs of the currently most loaded junctions in the network. In cases of large-scale networks, where regions exist with distinct traffic loads, TUC can be applied to each region separately; in which case each region may have its own adapted cycle time. Even in this case, however, there may be links that feature lower and strongly varying demand, which may lead to waste of green time, especially during off-peak periods. According to a recent study by Manolis et al. [12], during such periods, traffic regulation at the local junction level on a second-by-second basis and in a purely decentralized way may outperform TUC. It might thus be possible to mitigate green time waste and improve performance by enabling local second-by-second micro-regulation of the TUC signal plans to account for intra-cycle variations of the local traffic conditions.

Micro-regulation or traffic actuation, as commonly called, is a functionality offered at the local junction level by modern controllers. When enabled, traffic actuation modifies the background implemented signal plan in response to the detected local traffic conditions. This way, stages of the signal cycle with higher demands may take advantage of the unused green time at stages with less demand, which can be beneficial, as it reduces the possibility of green time waste. When applied, however, on coordinated signal plans, traffic actuation may disturb the offsets, since the different signal cycle stages may start earlier than defined due to lack of demand for previous stages. The offset disturbance on its turn may disturb traffic progression along the network arterials, thus creating stops and delays that would have been avoided if offsets were respected. There is therefore a known trade-off among the benefits and negative impacts of coordinated actuated signal control.

It is the aim of this paper to investigate, with simulation means, the aforementioned trade-off and to develop and examine different options for traffic actuation of the TUC-decided signal plans so that benefits are exploited and negative impacts are reduced to the maximum possible extent. For performance assessment, the resulting coordinated actuated control schemes are compared with TUC without traffic actuation using the AIMSUN simulation model of the city center of Chania, Greece.

\section{The TUC strategy}

The TUC strategy was developed so as to provide coordinated traffic responsive control in large-scale urban networks. This objective is approached using appropriate methodological tools, which allow for efficient application to large-scale networks and give rise to characteristics such as [11]:

- robustness with respect to measurement inaccuracies, disturbances, and hardware failures (detectors, communication links, etc.);

- applicability to networks of arbitrary characteristics and dimensions;

- simplicity of design and implementation code;

- limited measurement (one detector per link, arbitrary detector location within links) and communication (measurements or decisions once per cycle) requirements; and

- low computational effort.

TUC comprises three distinct but interconnected modules [11]. The two of them, following a centralized design approach, allow for the control of green splits and cycle time in real time, while the third, using a decentralized feedback control law, specifies the offsets of the main stages of successive junctions along arterials to create green waves, taking into account the possible existence of vehicle queues in the links.

In the original TUC strategy $[5,11]$, for the control of green splits, a multivariable regulator is used that modifies 
given fixed-time plans based on the currently observed traffic loads in the network links. In case of oversaturated conditions, the sensitivity of the regulator to the particular utilized fixed plans is minor. When demands and queueing, however, are low, TUC's split decisions are close to the utilized fixed plans. Thus, its performance depends on the quality of these plans, which need to be the appropriate for the considered traffic load. To circumvent the need for good fixed-time plans, the hybrid variant of the TUC strategy was developed, whereby signalized junctions are controlled by a real-time Webster-type demand-driven strategy as long as traffic conditions are undersaturated; while a switching to the original TUC is effectuated when traffic conditions are close to saturation [13]. For the needs of the present study, the hybrid variant of TUC is used, which has been found to be efficient during both off-peak and congested traffic conditions.

\section{Traffic actuation of TUC coordinated control decisions}

Traffic actuation is implemented via allowing traffic controllers to rotate signals from stage to stage in the cycle, based on current local second-by-second traffic-related information and user-defined signal timing parameters. To this end, detector loops are usually used, which send messages to the controllers as vehicles approach a junction. Messages concern, among others, vehicle presence and/or passage and time gaps in traffic flow, which the controllers use to call a stage in presence of demand, extend a stage to serve arriving demand or clear an existing queue, terminate or even skip a stage in case of lack of demand, provide safe stage termination for high-speed vehicle movements, etc. [14].

In a fully actuated control scheme, each stage runs from a minimum up to a maximum green time, if sufficient demand is present (max out operation), or terminates earlier (gap out operation) in case of a sufficient gap time in demand [14]. The minimum green time represents the least amount of time that a green signal indication will be displayed for a stage, while the maximum green time represents the maximum amount of time that a green signal indication can be displayed. Gap time is a parameter used to terminate the current stage, if actuated, when a gap in traffic is identified; vehicle calls extend green time of the current stage until a gap in detector occupancy is identified that is higher than the assigned gap time. In case of complete lack of demand, stages can also be omitted in a fully actuated control scheme.

In a coordinated actuated control scheme, on the other hand, all junctions need to have the same cycle time so as to maintain a consistent time-based relationship among them. In order to preserve this common cycle time, stages are divided in coordinated and actuated [14]. Actuated stages run in the same way that stages run in the fully actuated scheme, while coordinated stages run in a way that retains constant the so-called offset reference points. Offset reference points help structure the relationship among coordinated junctions by defining the point in time when the cycle begins timing at each junction. Offset reference points can be defined [14] using a variety of points in a cycle, such as e.g. the start of the green time of a coordinated stage or the start of the yellow time, that is the end of the green time of the stage. Usually, the offset reference point coincides with the end of the green time of the coordinated stage, which is observable and constant, in contrast to the start of green time, which may change due to gap outs of preceding actuated stages.

As far as TUC is concerned, its offset control module specifies at each junction the start of the green time of a single stage, defined to be the main stage of the junction. The main TUC stages are those that usually serve the heavier traffic loads of their respective junctions. They are also considered to be the most significant stages with respect to the creation of green waves along the network arterials. Given this context, several alternative options may be envisaged for local traffic actuation in a TUC-based coordinated actuated control scheme, such as:

- The main stages of TUC are defined as coordinated stages. With this specification, the main stages of TUC benefit of any unused green time from preceding actuated stages, while being prevented from actuation and potential gap outs, which could harm not only the local traffic conditions, but the conditions in the network as a whole. In this case, however, the TUCspecified offsets may be disturbed as a result of early green start due to insufficient demands leading to gap outs of the preceding actuated stages.

- One could define as coordinated stage at each junction, the stage that precedes the main stage of TUC. This approach preserves the TUCspecified offset (i.e. the start of green time of the main stage), but then the main stage cannot benefit of any spare green time from gap outs of the preceding actuated stages, since only the stage that is defined as coordinated takes advantage of any spare green time [15].

- Other stages could be specified as coordinated stages, with according advantages and disadvantages.

The above discussion makes clear that there is no unique and straightforward way towards a TUC-coordinated actuated control scheme, hence several alternative configurations, each carrying both benefits and shortcomings, which need to be carefully studied. As a basis for alternative configurations, the hybrid-TUC version is used. The green times decided by TUC are used as maximum green times for the actuated stages, while for minimum green times, the minimum green times also considered in TUC can be used. 
Note that stage skipping is not considered here, although it is an option worth to investigate in the future.

Given the aforementioned basic features, four cases, A, $\mathrm{B}, \mathrm{C}$ and $\mathrm{D}$ are examined as alternative TUC-coordinated actuated control configurations. The features of these cases are described below.

According to case $A$, at each junction, the main TUC stage is defined to be the coordinated stage; all other stages are actuated. Offset reference point is thus the end of the green time of the main TUC stage. This implies that the main TUC stage takes advantage of all spare green time due to gap outs of the preceding actuated stages. This also implies, however, that the main stage may start earlier than ordered by TUC, thus disturbing the TUC-specified offsets. To avoid potentially harmful and/or undesired early gap outs of stages that according to historical data carry heavy loads, values higher than those considered by TUC may be used as minimum green times (e.g. $80 \%$ of maximum green times). Alternatively, a higher gap time could be considered for these stages.

According to case $B$, at each junction, the stage that precedes the main TUC stage is defined to be the coordinated one; all other stages, including the main TUC stage, remain actuated. Offset reference point is thus the start of the green time of the main TUC stage. This means that it starts as ordered by TUC, thus preserving the decided offsets; it does not however benefit at all from any spare green time due to gap outs of other actuated stages. Any spare green time is used by the stage that has been defined as coordinated. As in case A, above, to avoid early gap outs of the main TUC stage, as well as of any other actuated stage, values higher than those considered by TUC may be used as minimum green times (e.g. $80 \%$ of maximum green times) or higher gap times may be defined.

Case $C$ is the same like case B with one difference. Actuation is prohibited for the main stage of TUC. This means that both the start and the end of the green time of the main TUC stage remain as decided and ordered by TUC.

The advantage of configuration case $\mathrm{A}$ against both $\mathrm{B}$ and $\mathrm{C}$ is that any spare green time due to gap outs of actuated stages will be used by the main TUC stage, while the advantage of configuration cases $\mathrm{B}$ and $\mathrm{C}$ against $\mathrm{A}$ is that offsets are preserved. It is the aim of configuration case $D$ to combine as much as possible the advantages of cases A, B and C, while mitigating at the same time their shortcomings. To this end, at each junction, the stage preceding the main TUC stage is defined as coordinated. This way, offset reference point is the start of the green time of main TUC stage thus preserving the TUC-decided offsets like in cases B and C. Unlike however cases B and C, any spare green time of the actuated stages is temporarily stored and added at the end of the green time of the main TUC stage during the next cycle time. This way, the main stage of TUC can take some advantage of the spare green time like in case A.

To implement control scheme $\mathrm{D}$, the following procedure is pursued:

1. During the first implementation cycle, coordinated actuated control is applied just like case $\mathrm{C}$ above, keeping at the same time track of any unused green times due to gap outs of actuated stages.

2. At the start of the next cycle, the maximum green times of all but the coordinated stages are updated on the basis of their previous unused green times. More specifically, the maximum green time of the main TUC stage is increased by a value equal to the total unused green time of the previous cycle, while the maximum green times of the actuated stages are decreased by values equal to their respective unused green times; the updates are of course subject to any applicable minimum green time constraints.

3. While on next cycle, actuation is applied under the maximum green time constraints set up in step 2 , keeping again track of any unused green times due to gap outs of actuated stages.

4. Steps 2 and 3 are repeated as long as actuated stages gap out, while in case of max outs of actuated stages, the whole process returns and starts again from step 1.

A disadvantage of case $\mathrm{D}$ is that any stage changes are postponed until the next cycle, hence the second-by-second reaction of the actuation is not preserved.

\section{Simulation investigations and results}

\subsection{Simulation setup}

To study and compare the performance and efficiency of the four alternative TUC-coordinated actuated control configurations presented and discussed in Section 3 under realistic traffic conditions, the road network of the city center of Chania, Greece, has been used (see Fig. 1). This network, which suffers from severe congestion problems leading sometimes even to gridlocks, was simulated in AIMSUN, a well-known microscopic traffic flow simulation software tool.

The network under study consists of 13 junctions, operating under signal cycles of common length divided in 2 to 4 stages, and 47 links. Each link is considered to be equipped with two spot detectors:

- one detector located around the middle of the link (as in the real network), which is used for the needs of the TUC strategy, and 


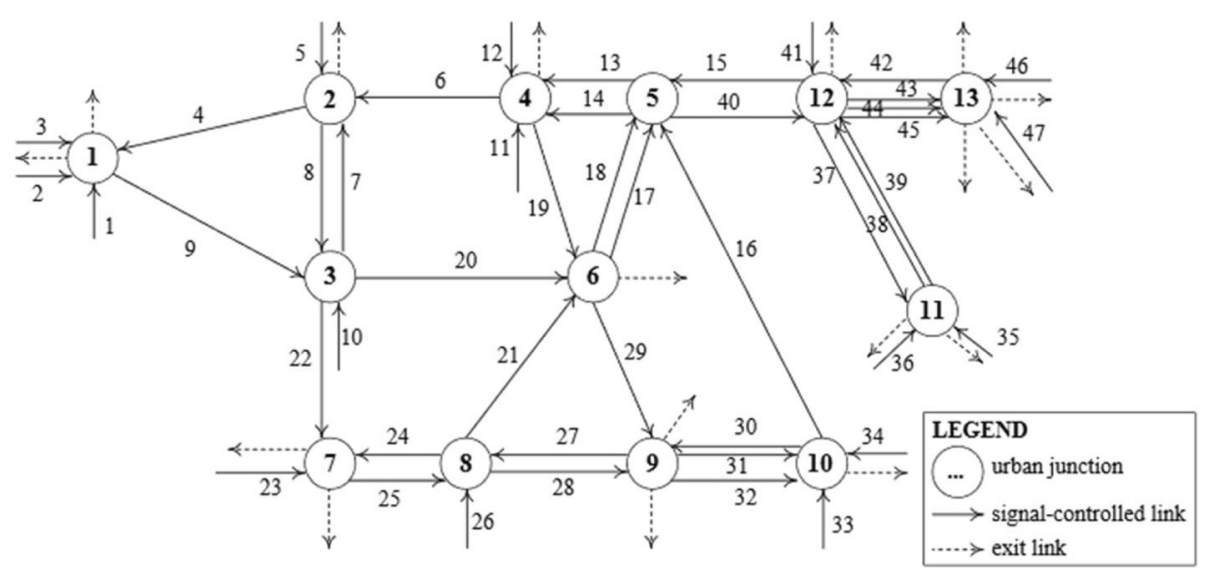

Fig. 1 The urban network of the city center of Chania, Greece

- one detector located $30 \mathrm{~m}$ upstream the stopline, which is used for actuation purposes.

The latter detectors have been located at $30 \mathrm{~m}$ in order to create a gap time parameter of $2 \mathrm{~s}$. After some experimentation with the Chania urban network, it was found out that, due to low demands, actuated stages serving secondary movements were running most times at or close to their minimum green times. As a consequence, gap times higher than $2 \mathrm{~s}$ for the respective secondary movements were letting no room at all for actuation, and were thus indifferent for their own traffic conditions and the traffic conditions of the whole network, as well.

As far as the rest of the parameters, required for applying TUC-coordinated actuated control, are concerned, the following have been considered:

- Control constraints such as minimum green times and minimum/maximum cycle times, etc. were suggested by the system operators of the Traffic Control Center (TCC) of Chania during a past study [13].

- When applying configuration case A, the minimum green time of the actuated stage at junction 7 is taken equal to $80 \%$ of its maximum green time. This particular junction has two stages only, both carrying heavy loads. As a consequence, early gap out of any of its stages may trigger congestion problems that can easily spread to the rest of the network.

- When applying configuration case B, where the main TUC stages are actuated, their minimum green times are taken equal to $80 \%$ of their maximum green times to avoid potentially harmful early gap outs.

To enable testing of the alternative TUC-coordinated actuated control configurations under different traffic conditions in the network, three different scenarios were developed, which were designed based on actual measurements to reflect the real traffic phenomena in the network under medium and high demand; see also Kouvelas et al. [13]. The three demand scenarios, each of them having a $1.5 \mathrm{~h}$ simulation horizon, have the following characteristics:

- Demand scenario 1: Light traffic conditions; involves a network-wide total demand that reaches the level of $4000 \mathrm{veh} / \mathrm{h}$ in the peak period.

- Demand scenario 2: Dense traffic conditions; involves a network-wide total demand that reaches the level of $4850 \mathrm{veh} / \mathrm{h}$ in the peak period.

- Demand scenario 3: Congested traffic conditions; involves a network-wide total demand that reaches the level of $5650 \mathrm{veh} / \mathrm{h}$ in the peak period.

All aforementioned scenarios involve a trapezoidal demand pattern. More specifically, in all scenarios, demands increase gradually from zero, leading eventually to peak periods starting around the 30th minute of the simulation horizon. Peak periods last for approximately $30 \mathrm{~min}$. Then, for the next $20 \mathrm{~min}$, demands reduce gradually to zero and remain zero for the last $10 \mathrm{~min}$ of the simulation. This way, in all scenarios, the network is empty at the end of the simulation horizon, which ensures a fair comparison of the alternative control configurations, as all arriving demand is being served by all control approaches examined.

The simulation step for the microscopic simulation model of the considered network has been set to $1 \mathrm{~s}$, while the traffic network characteristics (e.g., saturation flows and turning rates) were also suggested by the TCC operators during a past study [13].

The simulation results have been summarized in terms of the following AIMSUN-calculated performance indices: 
- Delay time: Average delay time per vehicle per kilometer $(\mathrm{s} / \mathrm{km})$.

- Mean speed: Mean network speed $(\mathrm{km} / \mathrm{h})$.

- Number of stops: Average number of stops per vehicle per kilometer (stops $/ \mathrm{km}$ ).

These performance indices are calculated as averages of the corresponding results of 10 simulation replications with different seeds to mitigate the effects of demand and simulator stochasticity.

\subsection{Simulation results and discussion}

Tables 1, 2 and 3 summarize the simulation results of demand scenarios $1-3$ in terms of the AIMSUN calculated performance indices mentioned in Section 4.1. According to these results, in case of demand scenario 1 (see Table 1), changes, either positive or negative, seem rather insignificant, as however expected. In this particular scenario, the relatively low demands create traffic conditions in the network, which are best served via low cycle times, and consequently low green times. More specifically, in this particular demand scenario, TUC operates all junctions mostly with the minimum cycle time that is $60 \mathrm{~s}$, and, almost all actuated stages with minimum green times equal to $7 \mathrm{~s}$. As a consequence, there is not much room for gap outs of the actuated stages, and the green time that remains unused is rather negligible to make any statistically significant difference.

On the other hand, in demand scenario 3 (see Table 3), the high demands create, for the major part of the simulation horizon, such high congestion levels all around the network, that actuated stages mostly max out. Gap outs of the actuated stages take thus place mainly at the early and last steps of the simulation horizon, i.e. before the onset and after the end of peak period. During these two periods, some green time remains unused from the actuated stages and is relocated to others. That is why in most of the considered coordinated actuated control configurations, some small improvement is observed in all performance indices (see Table 3). More specifically, all but configuration case B present some improvement indications compared to TUC.
In case of configuration $\mathrm{B}$, the high traffic loads in combination with the fact that the main TUC stages are (even with increased minimum green times) actuated and at the same time do not benefit from any unused green times, create negative effects for the traffic conditions all over the network. That is, after all, why control configuration case $\mathrm{B}$ presents the worst performance from all other considered coordinated actuated control cases in all considered demand scenarios. Its performance is even worse than TUC in all but demand scenario 2 , where actuation indicates some, though very small, improvement.

In general, in demand scenario 2, all considered coordinated actuated control configurations seem to perform better than TUC (see Table 2). The observed improvements, however, seem rather negligible, especially under control configuration cases $\mathrm{B}$ and $\mathrm{C}$. Figure 2 gives a relevant example based on junction 10. According to this figure, stage 1 greatly disbenefits under configuration $\mathrm{B}$, although it serves the needs of main streets. This stage is the main TUC stage of the junction, but, in case $\mathrm{B}$, in order to preserve the TUC-decided offsets, stage 3 is defined as the coordinated stage, while stage 1 is defined as actuated along with stage 2 . As a consequence, for most part of the simulation horizon, the green time trajectory of stage 1 remains with configuration $\mathrm{B}$ constantly below the green time trajectories of all other considered configurations. The opposite behavior is observed in stage 3; its green time trajectory is constantly above the green time trajectories of all other configurations for most part of the simulation horizon, as it takes advantage of all unused green times. The unused green times, however, which are relocated to stage 3 , come from stages 1 and 2; and, while stage 2 is not affected, as it indeed serves the needs of a secondary street with low traffic demand, stage 1 is seriously harmed.

Generally, TUC-coordinated actuated control configuration A delivers improvements compared to TUC, which are statistically significant in all examined demand scenarios, while configuration B creates, as discussed earlier, disbenefits rather than benefits. On the other hand, configuration $\mathrm{C}$ seems to have minor effects at least in the case of the Chania network where all,

Table 1 Simulation results for demand scenario 1 (light traffic conditions)

\begin{tabular}{|c|c|c|c|c|c|c|}
\hline \multirow{2}{*}{$\begin{array}{l}\text { Control } \\
\text { configuration }\end{array}$} & \multicolumn{2}{|l|}{ Delay } & \multicolumn{2}{|c|}{ Mean network speed } & \multicolumn{2}{|c|}{ Number of stops } \\
\hline & $\begin{array}{l}\text { Average } \\
\text { value }\end{array}$ & $\begin{array}{l}\% \text { change compared to } \\
\text { TUC }\end{array}$ & $\begin{array}{l}\text { Average } \\
\text { value }\end{array}$ & $\begin{array}{l}\% \text { change compared to } \\
\text { TUC }\end{array}$ & $\begin{array}{l}\text { Average } \\
\text { value }\end{array}$ & $\begin{array}{l}\% \text { change compared to } \\
\text { TUC }\end{array}$ \\
\hline TUC & 72.40 & - & 25.74 & - & 2.97 & - \\
\hline Case A & 71.40 & -1.38 & 25.92 & +0.73 & 2.94 & -1.12 \\
\hline Case B & 73.66 & +1.74 & 25.50 & -0.90 & 3.00 & +0.86 \\
\hline Case C & 72.74 & +0.47 & 25.68 & -0.23 & 2.99 & +0.44 \\
\hline Case D & 72.91 & +0.70 & 25.65 & -0.35 & 2.99 & +0.63 \\
\hline
\end{tabular}


Table 2 Simulation results for demand scenario 2 (dense traffic conditions)

\begin{tabular}{|c|c|c|c|c|c|c|}
\hline \multirow{2}{*}{$\begin{array}{l}\text { Control } \\
\text { configuration }\end{array}$} & \multicolumn{2}{|l|}{ Delay } & \multicolumn{2}{|c|}{ Mean network speed } & \multicolumn{2}{|c|}{ Number of stops } \\
\hline & $\begin{array}{l}\text { Average } \\
\text { value }\end{array}$ & $\begin{array}{l}\text { \% change compared to } \\
\text { TUC }\end{array}$ & $\begin{array}{l}\text { Average } \\
\text { value }\end{array}$ & $\begin{array}{l}\text { \% change compared to } \\
\text { TUC }\end{array}$ & $\begin{array}{l}\text { Average } \\
\text { value }\end{array}$ & $\begin{array}{l}\% \text { change compared to } \\
\text { TUC }\end{array}$ \\
\hline TUC & 85.96 & - & 23.47 & - & 3.28 & - \\
\hline Case A & 81.41 & -5.29 & 24.18 & +3.07 & 3.15 & -4.00 \\
\hline Case B & 85.64 & -0.37 & 23.52 & +0.21 & 3.26 & -0.84 \\
\hline Case C & 85.56 & -0.46 & 23.53 & +0.26 & 3.28 & -0.22 \\
\hline Case D & 84.08 & -2.19 & 23.77 & +1.28 & 3.21 & -2.20 \\
\hline
\end{tabular}

actuated under this case, stages serve in fact secondary streets with low demands. As a consequence, they are operated by TUC mostly at their minimum green times, thus leaving not much space for gap outs and spare green time that could be used to serve the needs of the main streets. Configuration D on the other hand, seems to suffer from the late (often outdated) application of the actuation decisions, as well as from the fact that a stage that is not the main TUC stage is defined as coordinated, which means that it will use all its TUC-decided green time no matter its actual demand needs. Thus this configuration delivers mixed results.

Figure 3 gives an example of the reasons behind the limited effects that TUC-coordinated actuated control configuration D eventually has on the Chania network. The figure compares the green times resulting for the stages of junction 1 when operating under TUC and under configuration D. In this particular junction, stage 1 is the main TUC stage, thus stage 3 becomes the coordinated, and stage 2 remains the only actuated stage of the junction. As however, displayed in the figure, TUC runs the actuated stage 2 for most of the time at minimum green time. As a consequence, stage 2 rarely terminates earlier so as to leave unused green time for relocation to stage 1 . It is only at the first and last part of the simulation horizon that TUC decides for stage 2 green times higher than the minimum, but at these time periods, the demands of the other stages are not such that could really benefit from using the unused green time of stage 2 . In contrast to case $\mathrm{D}$, under case $\mathrm{A}$, both stages 2 and 3 run actuated, thus stage 1 benefits not only from the spare green time of stage 2 , but also from the spare green time of stage 3 , which runs actuated under this configuration and gaps out frequently. As the graph of stage 3 in Fig. 3 indicates, it is only during the peak period (cycle numbers 21 to 38 , approximately), that stage 3 uses all the green time allocated by TUC, while before the onset and after the end of the peak period (i.e. before cycle number 21 and after 38 ) it mostly runs at minimum green time.

\section{Conclusions}

The TUC strategy was developed aiming at coordinated traffic-responsive signal control in large-scale urban networks, and has demonstrated high efficiency especially under saturated traffic conditions [16]. TUC adopts one common cycle time for all network junctions at any time, which is computed considering the needs of the currently most loaded junctions in the network. In cases of large-scale networks, where regions exist with distinct traffic loads, TUC can be applied to each region separately; in which case, each region may have its own adaptive cycle time. Even in this case, however, there may be links that feature lower and strongly varying demands, which may lead to waste of green time, especially during off-peak periods. Such waste could be mitigated by enabling actuation of the TUC signal plans at the local junction level, thus accounting for the intra-cycle variations of the local traffic conditions.

Actuation of the TUC signal plans may be enabled under several configurations, four of which were developed and tested herein:

Table 3 Simulation results for demand scenario 3 (congested traffic conditions)

\begin{tabular}{|c|c|c|c|c|c|c|}
\hline \multirow{2}{*}{$\begin{array}{l}\text { Control } \\
\text { configuration }\end{array}$} & \multicolumn{2}{|l|}{ Delay } & \multicolumn{2}{|c|}{ Mean network speed } & \multicolumn{2}{|c|}{ Number of stops } \\
\hline & $\begin{array}{l}\text { Average } \\
\text { value }\end{array}$ & $\begin{array}{l}\text { \% change compared to } \\
\text { TUC }\end{array}$ & $\begin{array}{l}\text { Average } \\
\text { value }\end{array}$ & $\begin{array}{l}\text { \% change compared to } \\
\text { TUC }\end{array}$ & $\begin{array}{l}\text { Average } \\
\text { value }\end{array}$ & $\begin{array}{l}\% \text { change compared to } \\
\text { TUC }\end{array}$ \\
\hline TUC & 116.13 & - & 19.68 & - & 3.85 & - \\
\hline Case A & 112.65 & -3.00 & 20.05 & +1.84 & 3.76 & -2.51 \\
\hline Case B & 117.82 & +1.45 & 19.52 & -0.83 & 3.87 & +0.43 \\
\hline Case C & 115.68 & -0.40 & 19.73 & +0.23 & 3.84 & -0.33 \\
\hline Case D & 116.02 & -0.10 & 19.69 & +0.04 & 3.85 & -0.23 \\
\hline
\end{tabular}



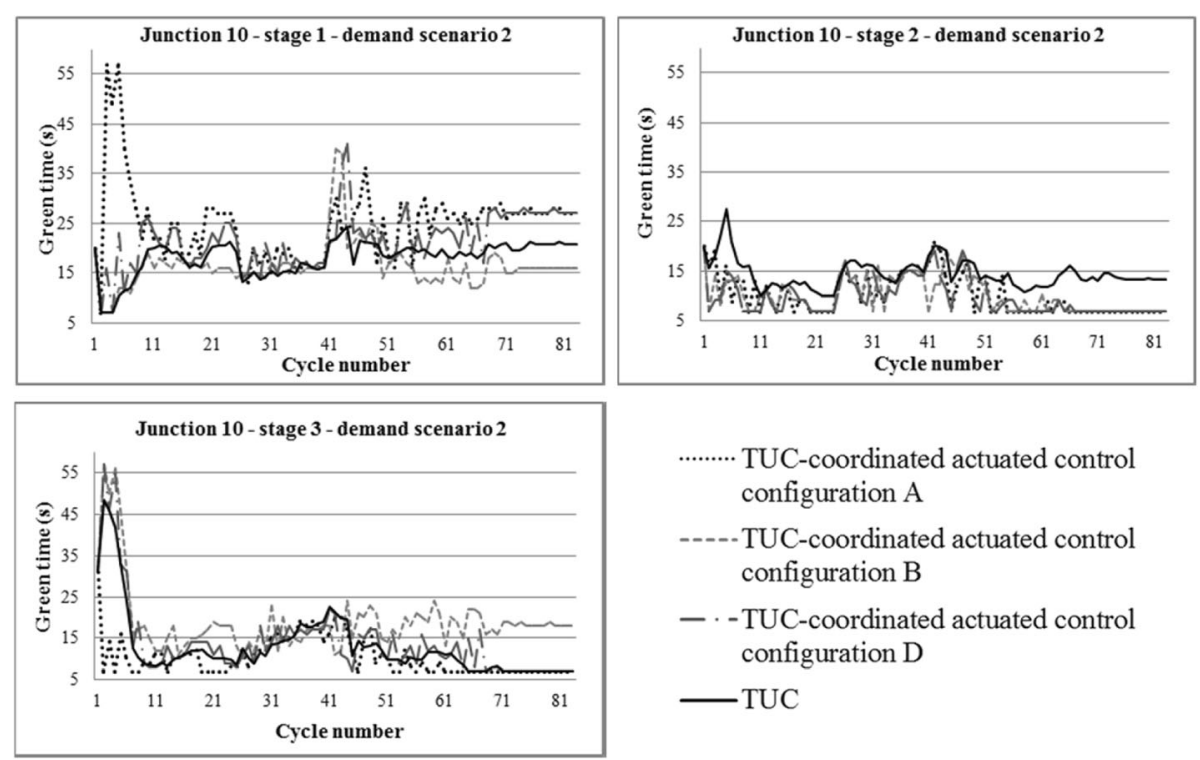

......... TUC-coordinated actuated control configuration A

-----TUC-coordinated actuated control configuration $\mathrm{B}$

- - - TUC-coordinated actuated control configuration D

- TUC

Fig. 2 Green times of TUC vs alternative TUC-coordinated actuated control configurations; stage 1 is the main TUC stage at junction 10

- Under TUC-coordinated actuated control configuration case $\mathrm{A}$, actuation is enabled so that all unused green time is relocated to the main TUC stages, i.e. the stages of signal control serving the major traffic movements.

- Under TUC-coordinated actuated control configuration cases $B$ and $C$ actuation is enabled so as to preserve the TUC-decided offsets; in case $B$, the main TUC stages run actuated, while in case C, actuation is prohibited for them.
- Under TUC-coordinated actuated control configuration case D, effort is put so as to relocate the unused green time to the main TUC stages and at the same time preserve the TUC-decided offsets.

Case A proved to be the best approach, but improvements over the TUC strategy were moderate; this may be a result of the signal control structure and settings of the particular network under study or the particular (trapezoidal) demand distribution considered in all examined demand scenarios.
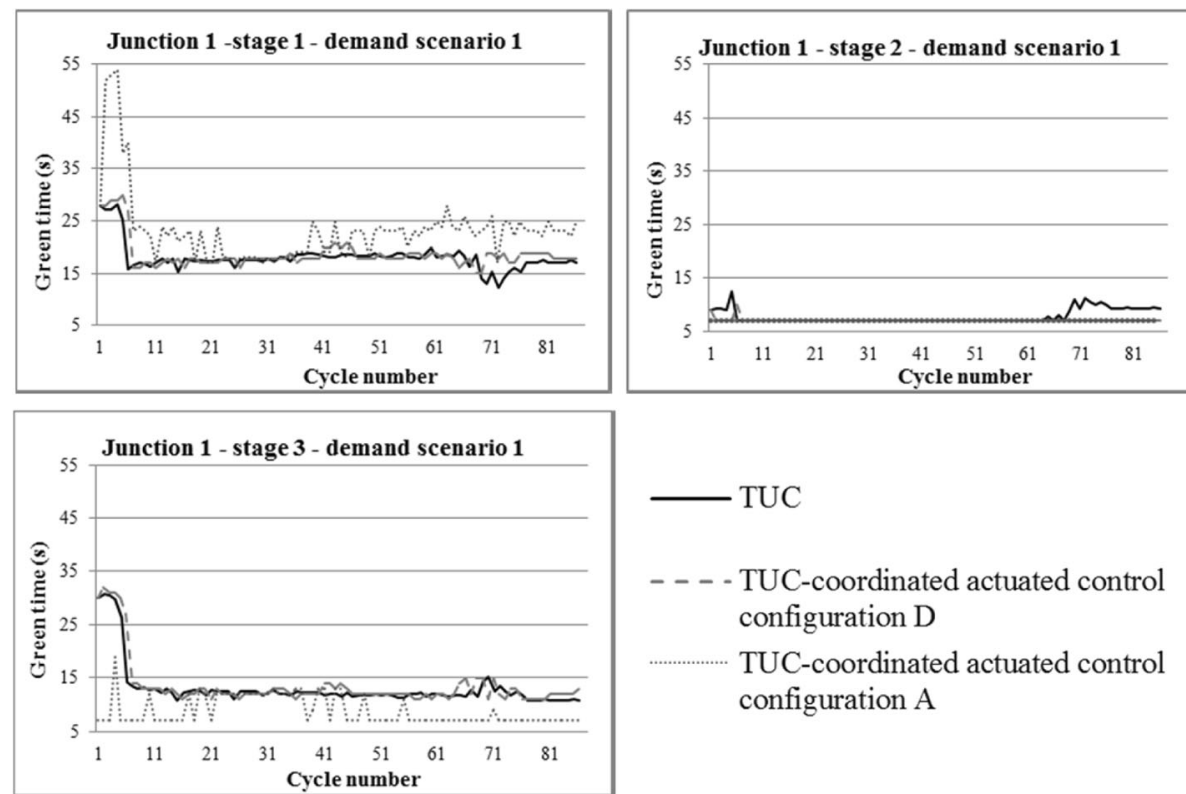

TUC

- - - - TUC-coordinated actuated control configuration D

TUC-coordinated actuated control configuration A

Fig. 3 Green times of TUC vs TUC-coordinated actuated control configuration cases A and D; stage 1 is the main TUC stage at junction 1 
Actuation is known to be most helpful in cases of asymmetric and strongly varying demand patterns, and this is an option that should be examined in the future. Stage skipping in case of lack of demand is another option that may under circumstances prove particularly useful, but was not considered in the investigations reported herein. Finally, further tests with other road networks are necessary before reaching final conclusions regarding the benefits and potential shortcomings of the alternative TUC-coordinated actuated control configurations.

\section{Authors' contributions}

All authors read and approved the final manuscript.

\section{Competing interests}

The authors declare that they have no competing interests.

\section{Publisher's Note}

Springer Nature remains neutral with regard to jurisdictional claims in published maps and institutional affiliations.

Received: 16 March 2018 Accepted: 4 June 2018

Published online: 13 June 2018

\section{References}

1. EC (2011) White paper, roadmap to a single European transport Area Towards a competitive and resource efficient transport system. European Union, Brussels http://eur-lex.europa.eu/legal-content/EN/TXT/PDF/?uri= CELEX:52011DC0144\&from=EN. Accessed 5 May 2018

2. EC (2016) The implementation of the 2011 White Paper on Transport "Roadmap to a Single European Transport Area - towards a competitive and resource-efficient transport system" five years after its publication: achievements and challenges. Commission Staff Working Document, European Union, Brussels https://ec.europa.eu/transport/sites/transport/files/ themes/strategies/doc/2011_white_paper/swd\%282016\%29226.pdf. Accessed 9 June 2018.

3. Papageorgiou M, Diakaki C, Dinopoulou V, Kotsialos A, Wang Y (2003) Review of road traffic control strategies. Proc IEEE 91(12):2043-2067

4. Gordon RL, Warren Tighe PE (2005) Traffic Control Systems Handbook. Report No. FHWA-HOP-06-006 prepared for the Office of Transportation Management, Federal Highway Administration. https://ops.fhwa.dot.gov/ publications/fhwahop06006/index.htm. Accessed 5 May 2018

5. Diakaki C, Papageorgiou M, Aboudolas K (2002) A multivariable regulator approach to traffic-responsive network-wide signal control. J Control Eng Pract 10:183-195

6. Diakaki C, Papageorgiou M, McLean T (2000) Application and evaluation of the integrated traffic-responsive urban corridor control strategy IN-TUC IN Glasgow. Transp Res Rec 1727:101-111

7. Dinopoulou V, Diakaki C, Papageorgiou M (2005) Application and evaluation of the signal traffic control strategy TUC in Chania. J Intell Transp Syst 9(3): 133-143

8. Kosmatopoulos E, Papageorgiou M, Bielefeldt C, Dinopoulou V, Morris R, Mueck J, Richards A, Weichenmeier F (2006) International comparative field evaluation of a traffic-responsive signal control strategy in three cities. Transp Res A Policy Pract 40(5):399-413

9. Kraus W Jr, de Souza FA, Carlson RC, Papageorgiou M, Dantas LD, Camponogara E, Kosmatopoulos E, Aboudolas K (2010) Cost effective realtime traffic signal control using the TUC strategy. IEEE Intell Transp Syst Mag 2(4):6-17

10. Manolis D, Papamichail I, Kosmatopoulos EB, Papageorgiou M (2016) Automated tuning of ITS management and control systems: results from real-life experiments. Transp Res C 66:119-135

11. Diakaki C, Dinopoulou V, Aboudolas K, Papageorgiou M, Ben-Shabat E, Seider E, Leibov A (2003) Extensions and new applications of the trafficresponsive urban control strategy: coordinated signal control for urban networks. Transp Res Rec 1856:202-211

12. Manolis D, Pappa T, Diakaki C, Papamichail I, Papageorgiou M (2017) Simulation study of centralized vs decentralized approaches to the signal control of large-scale urban networks. In: Compendium of Papers of 96th Annual Meeting of the Transp Res Board (TRB), January 8-12. National Academy of Sciences [US] Transportation Research Board, Washington D.C

13. Kouvelas A, Aboudolas K, Kosmatopoulos EB, Papageorgiou M (2011) Adaptive performance optimization for large-scale traffic control systems. IEEE Trans Intell Transp Syst 12(4):1434-1445

14. NCHRP (2015) Signal Timing Manual. National Cooperative Research Program (NCRP) Report 812, $2^{\text {nd }}$ Edition, Transportation Research Board (TRB). National Academy of Sciences, USA

15. Skabardonis A (1996) Determination of timings in signal systems with traffic-actuated controllers. Transp Res Rec 1554:18-26

16. Dinopoulou V, Diakaki C, Papageorgiou M (2006) Applications of the urban traffic control strategy TUC. Eur J Oper Res 175(3):1652-1665

\section{Submit your manuscript to a SpringerOpen ${ }^{\circ}$ journal and benefit from:}

- Convenient online submission

- Rigorous peer review

- Open access: articles freely available online

- High visibility within the field

- Retaining the copyright to your article

Submit your next manuscript at $\boldsymbol{\nabla}$ springeropen.com 